Open Access

\title{
Pulmonary ultrasound and pulse oximetry versus chest radiography and arterial blood gas analysis for the diagnosis of acute respiratory distress syndrome: a pilot study
}

Cameron M. Bass ${ }^{1,2^{*}}$ D, Dana R. Sajed ${ }^{1,2}$, Adeyinka A. Adedipe ${ }^{1}$ and T. Eoin West ${ }^{1,2}$

\begin{abstract}
Introduction: In low-resource settings it is not always possible to acquire the information required to diagnose acute respiratory distress syndrome (ARDS). Ultrasound and pulse oximetry, however, may be available in these settings. This study was designed to test whether pulmonary ultrasound and pulse oximetry could be used in place of traditional radiographic and oxygenation evaluation for ARDS.

Methods: This study was a prospective, single-center study in the ICU of Harborview Medical Center, a referral hospital in Seattle, Washington, USA. Bedside pulmonary ultrasound was performed on ICU patients receiving invasive mechanical ventilation. Pulse oximetric oxygen saturation $\left(\mathrm{SpO}_{2}\right)$, partial pressure of oxygen $\left(\mathrm{PaO}_{2}\right)$, fraction of inspired oxygen $\left(\mathrm{FiO}_{2}\right)$, provider diagnoses, and chest radiograph closest to time of ultrasound were recorded or interpreted.

Results: One hundred and twenty three ultrasound assessments were performed on 77 consecutively enrolled patients with respiratory failure. Oxygenation and radiographic criteria for ARDS were met in 35 assessments. Where $\mathrm{SpO}_{2} \leq 97 \%$, the Spearman rank correlation coefficient between $\mathrm{SpO}_{2} / \mathrm{FiO}_{2}$ and $\mathrm{PaO}_{2} / \mathrm{FiO}_{2}$ was $0.83, p<0.0001$. The sensitivity and specificity of the previously reported threshold of $\mathrm{SpO}_{2} / \mathrm{FiO}_{2} \leq 315$ for $\mathrm{PaO}_{2} / \mathrm{FiO}_{2} \leq 300$ was $83 \%$ (95\% confidence interval (Cl) 68-93), and $50 \%$ (95\% Cl 1-99), respectively. Sensitivity and specificity of $\mathrm{SpO}_{2} /$ $\mathrm{FiO}_{2} \leq 235$ for $\mathrm{PaO}_{2} / \mathrm{FiO}_{2} \leq 200$ was $70 \%(95 \% \mathrm{Cl} 47-87)$, and $90 \%$ (95\% Cl 68-99), respectively. For pulmonary ultrasound assessments interpreted by the study physician, the sensitivity and specificity of ultrasound interstitial syndrome bilaterally and involving at least three lung fields were $80 \%$ (95 \% Cl 63-92) and $62 \%$ (95 \% Cl 49-74) for radiographic criteria for ARDS. Combining $\mathrm{SpO}_{2} / \mathrm{FiO}_{2}$ with ultrasound to determine oxygenation and radiographic criteria for ARDS, the sensitivity was $83 \%$ (95\% Cl 52-98) and specificity was $62 \%$ (95\% Cl 38-82). For moderate-severe ARDS criteria $\left(\mathrm{PaO}_{2} / \mathrm{FiO}_{2} \leq 200\right)$, sensitivity was $64 \%(95 \% \mathrm{Cl} 31-89)$ and specificity was $86 \%$ (95\% Cl 65-97). Excluding repeat assessments and independent interpretation of ultrasound images did not significantly alter the sensitivity measures.
\end{abstract}

Conclusions: Pulse oximetry and pulmonary ultrasound may be useful tools to screen for, or rule out, impaired oxygenation or lung abnormalities consistent with ARDS in under-resourced settings where arterial blood gas testing and chest radiography are not readily available.

\footnotetext{
*Correspondence: cbass@alum.mit.edu

'Department of Medicine, University of Washington School of Medicine, Seattle, WA, USA

${ }^{2}$ International Respiratory and Severe IIIness Center, University of Washington School of Medicine, Seattle, WA, USA
}

\section{Biomed Central}

(c) 2015 Bass et al. This is an Open Access article distributed under the terms of the Creative Commons Attribution License (http://creativecommons.org/licenses/by/4.0), which permits unrestricted use, distribution, and reproduction in any medium, provided the original work is properly credited. The Creative Commons Public Domain Dedication waiver (http:// creativecommons.org/publicdomain/zero/1.0/) applies to the data made available in this article, unless otherwise stated. 


\section{Introduction}

Acute respiratory distress syndrome (ARDS) is a common cause of mortality in the ICU [1]. The diagnosis of ARDS is established using the new Berlin criteria which consists of four elements: 1) onset within a week of a known clinical insult or new respiratory symptoms, 2) bilateral opacities on chest radiograph or computed tomography scan, 3) respiratory failure not fully explained by cardiac failure or fluid overload, and 4) impaired oxygenation defined as partial pressure of oxygen $\left(\mathrm{PaO}_{2}\right)$ /fraction of inspired oxygen $\left(\mathrm{FiO}_{2}\right) \leq 300$ on positive end-expiratory pressure (PEEP) or continuous positive airway pressure (CPAP) $\geq 5$ $\mathrm{cmH}_{2} \mathrm{O}$. Diagnosis of ARDS requires an arterial blood gas (ABG) test and chest radiography or computed tomography in the appropriate clinical scenario.

In much of the world where medical resources are limited, blood gas analysis and imaging technologies may not be available, impairing the ability to make the diagnosis of ARDS. In one study, half of all patients who clinically had ARDS in a hospital in Rwanda had a chest radiograph available for review [2]. However, both pulse oximetry and ultrasound are becoming increasingly accessible worldwide [3, 4]. The pulse oximetric saturation to inspired oxygen ratio $\left(\mathrm{SpO}_{2} / \mathrm{FiO}_{2}\right)$ has been correlated with the $\mathrm{PaO}_{2} / \mathrm{FiO}_{2}$ ratio in ARDS $[5,6]$. Pulmonary ultrasound is a rapidly developing technology in which the diagnosis of lung disease is being explored in diverse settings, and new diagnostic criteria are being developed for multiple pulmonary processes [7]. Some of the first patterns of pulmonary ultrasound to be recognized were the distinct "A line" and "B line" artifacts [8]. The "A-line" pattern, characterized by horizontal reflection artifacts of the pleural line deep into the lung, is seen with alveoli that are physiologically filled with air. The "Bline" pattern, characterized by the presence of three or more vertical artifacts obliterating any A-lines, correlates with the ultrasound interstitial syndrome (UIS) [9]. The presence of UIS diffusely on ultrasound is considered consistent with either cardiogenic pulmonary edema or ARDS [10-12]. The A line and B line patterns have proven to be easily distinguished by a bedside clinician after relatively brief teaching $[13,14]$. Therefore, it is conceivable that the diagnosis of ARDS could be made using pulse oximetry and pulmonary ultrasound at the point of care.

We hypothesized that data derived from pulse oximetry and bedside pulmonary ultrasound could be used in lieu of ABG and chest radiography to meet oxygenation and radiographic criteria for ARDS. We designed a prospective study in patients with respiratory failure in the ICU to test this hypothesis.

\section{Methods}

\section{Study procedures}

The study was conducted in the ICUs at Harborview Medical Center, Seattle, Washington, USA. The study physician received $4 \mathrm{~h}$ of hands-on pulmonary ultrasound training from an ultrasound fellowship-trained emergency medicine attending physician. Training included ultrasound scanning and discussions at the bedside of ten patients with different lung pathologies and a brief literature review.

From 4 July to 22 August 2013, mechanically ventilated patients in the ICUs were identified by the study physician early each morning using a Quality Safety Dashboard, Monday through Friday. Patients were screened for study eligibility based on inclusion and exclusion criteria (Fig. 1). Although the study design initially included patients on high levels of supplemental oxygen via high flow nasal cannula or face mask, the revised Berlin definition of ARDS published in 2012 required administration of CPAP or PEEP, so eligibility was restricted to patients receiving mechanical ventilation. No attempts were made to determine the etiology or management of respiratory failure before enrollment. Contraindications included burns over the chest, flail chest, active hemodynamic instability or declination by the patient's nurse, receiving palliative care, age less than 18 years, incarceration, prone positioning, planned extubation the morning of study, or lack of identifying personal information.

For patients who met eligibility criteria the study physician performed an ultrasound evaluation prior to reviewing the patient's chart or other imaging. A Sonosite S-ICU ultrasound machine (SonoSite, Inc., Bothell, Washington) with a p21x 5-1 Mhz phased array probe was used to capture 6-second video imaging of the lung at three sites on each side of the chest (Fig. 2), following a modification of a previously described protocol $[10,15,16]$. Specifically, the six "BLUE" points were evaluated for the presence of B lines (Fig. 3). In order to mimic rapid evaluation by a single clinician in austere conditions, patients were not repositioned for the purpose of the scan and assistance from additional staff members was not requested. No scan was permitted to take more than $5 \mathrm{~min}$, including start up time for the machine and recording time for the videos. The $\mathrm{FiO}_{2}$ and $\mathrm{SpO}_{2}$ at the time of the ultrasound were recorded. This procedure was performed as close to $06: 30 \mathrm{am}$ as possible to reduce the duration between ultrasound and early morning chest radiographs and $A B G$ measurements performed in the ICUs. Subsequently, the chest radiograph and ABG performed closest to the time of the ultrasound were abstracted from the medical record. The $\mathrm{FiO}_{2}$ and $\mathrm{SpO}_{2}$ recorded by transcutaneous probe at the time of the $\mathrm{ABG}$ were also recorded. The ICU teams clinical note for the day of the study evaluation was reviewed to capture active clinical diagnoses. If patients continued to meet eligibility criteria, repeat study assessments were performed every 3 days. 


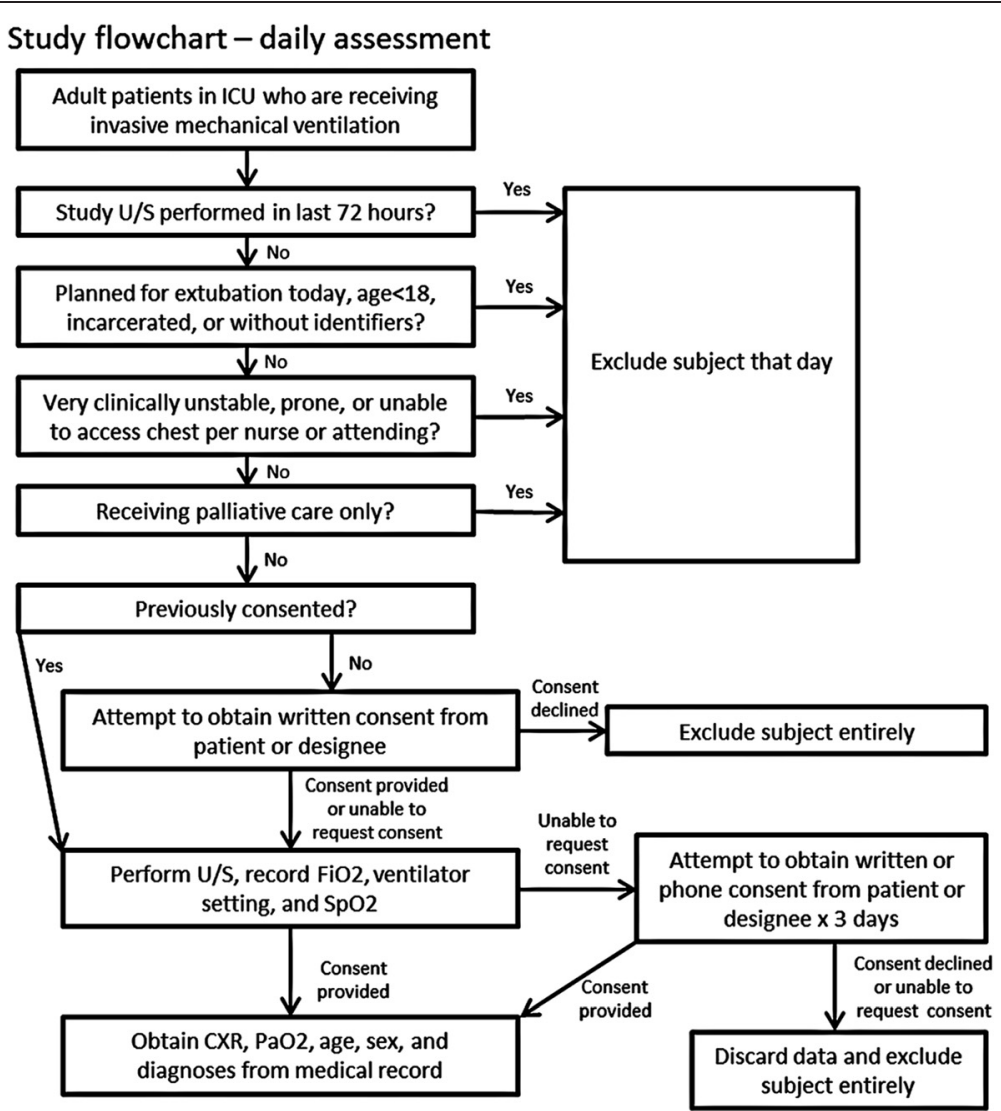

Fig. 1 Study flow chart. CXR chest x-ray, FiO2 fraction of inspired oxygen, PaO2 partial pressure of oxygen, SpO2, pulse oximetric oxygen saturation, U/S ultrasound

\section{Definitions}

For each study assessment, the ABG and concurrent $\mathrm{FiO}_{2}$ data was used to calculate a $\mathrm{PaO}_{2} / \mathrm{FiO}_{2}$ value. Chest radiographs were independently reviewed by a board-certified attending chest radiologist to determine if they met radiographic criteria for ARDS, namely bilateral opacities not fully explained by effusions, lobar/lung collapse, or nodules [17]. Computerized tomography scans of the chest were not available for all patients, so this modality was not incorporated into the study design. The radiologist was blinded to all other clinical information. Study assessments with $\mathrm{PaO}_{2} / \mathrm{FiO}_{2} \leq 300$ and a chest radiograph interpreted as consistent with ARDS were deemed to have met oxygenation and radiographic criteria for ARDS [17]. $\mathrm{PaO}_{2} / \mathrm{FiO}_{2} \leq 200$ was considered at least moderately impaired oxygenation.

For each study assessment the $\mathrm{SpO}_{2} / \mathrm{FiO}_{2}$ ratio was calculated using $\mathrm{SpO}_{2}$ and $\mathrm{FiO}_{2}$ recorded at the time of the ABG. Based on the relationship between $\mathrm{SpO}_{2} / \mathrm{FiO}_{2}$ and $\mathrm{PaO}_{2} / \mathrm{FiO}_{2}$ in ARDS patients derived by Rice et al. [5], $\mathrm{SpO}_{2} / \mathrm{FiO}_{2} \leq 315$ was considered impaired oxygenation and $\mathrm{SpO}_{2} / \mathrm{FiO}_{2} \leq 235$ at least moderately impaired oxygenation. UIS was defined as at least three B lines in a single frozen frame in one or more lung fields. Ultrasound imaging consistent with ARDS was determined by the presence of UIS bilaterally [7, 10, 12, 15]. Images that were difficult to interpret due to subcutaneous emphysema, obesity, consolidations, effusions, image quality or positioning were noted, but were still designated as consistent with UIS or not. All ultrasound video clips were batch reviewed and classified by the study physician and independently reviewed and classified by an ultrasound-trained attending physician in a blinded manner after the completion of patient enrollment.

\section{Statistics}

Continuous data were displayed as mean \pm standard deviation if normally distributed or median and interquartile range (IQR) if non-normally distributed. Correlation between continuous variables was determined using Spearman's rank correlation coefficient. Diagnostic accuracy measures of sensitivity and specificity were calculated with $95 \%$ exact binomial confidence intervals (CIs). Interobserver agreement was determined using the kappa coefficient. Nonparametric receiver operating curve analysis was used to determine area under the 

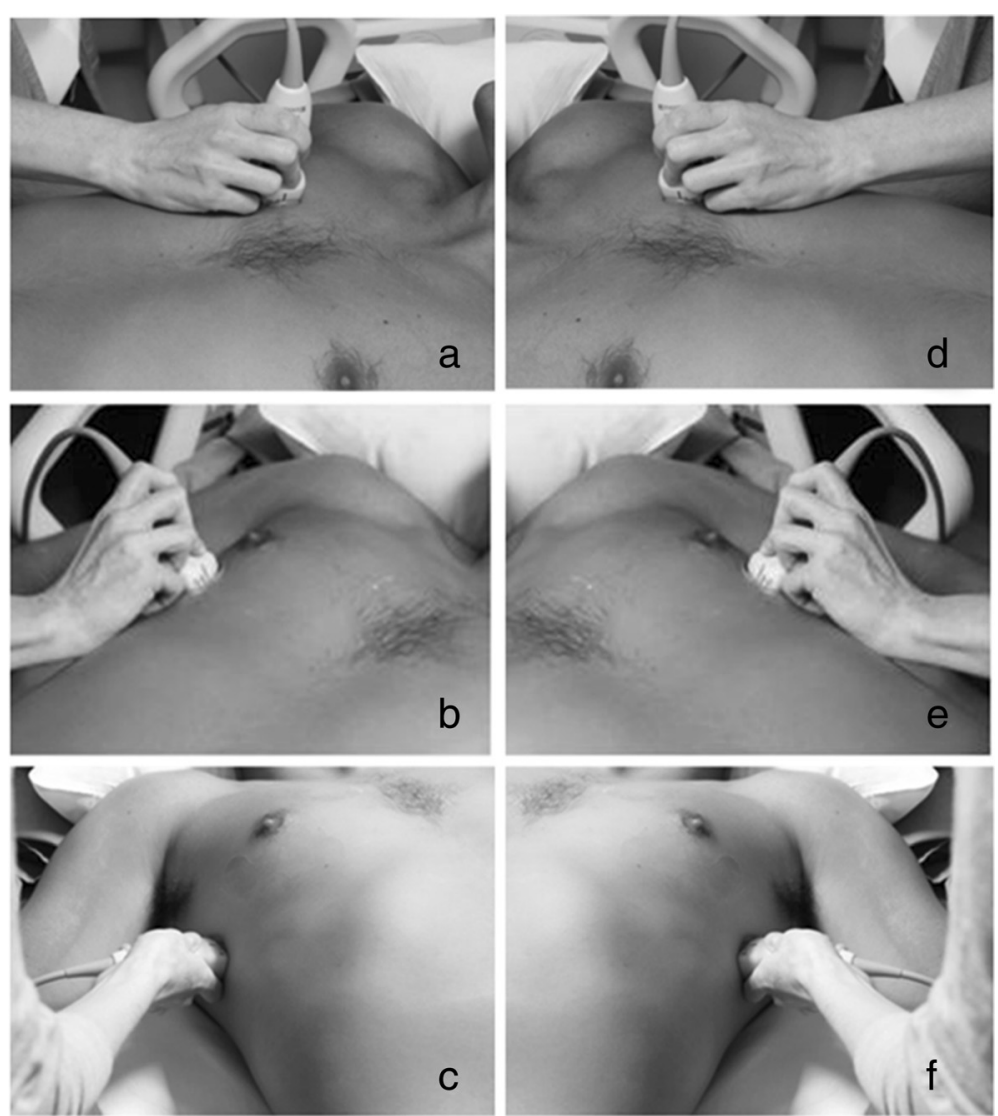

Fig. 2 Placement of the ultrasound probe at six locations on the chest. a zone 1 is $2 \mathrm{~cm}$ below the anterior mid-clavicular line on the right side of the chest; $\mathbf{b}$ zone 2 is $4 \mathrm{~cm}$ inferior and $4 \mathrm{~cm}$ lateral to zone 1; c zone 3 is $2 \mathrm{~cm}$ inferior to zone 2 along the mid-axillary line. $\mathbf{d}$-f The identical positions on the left side of the chest

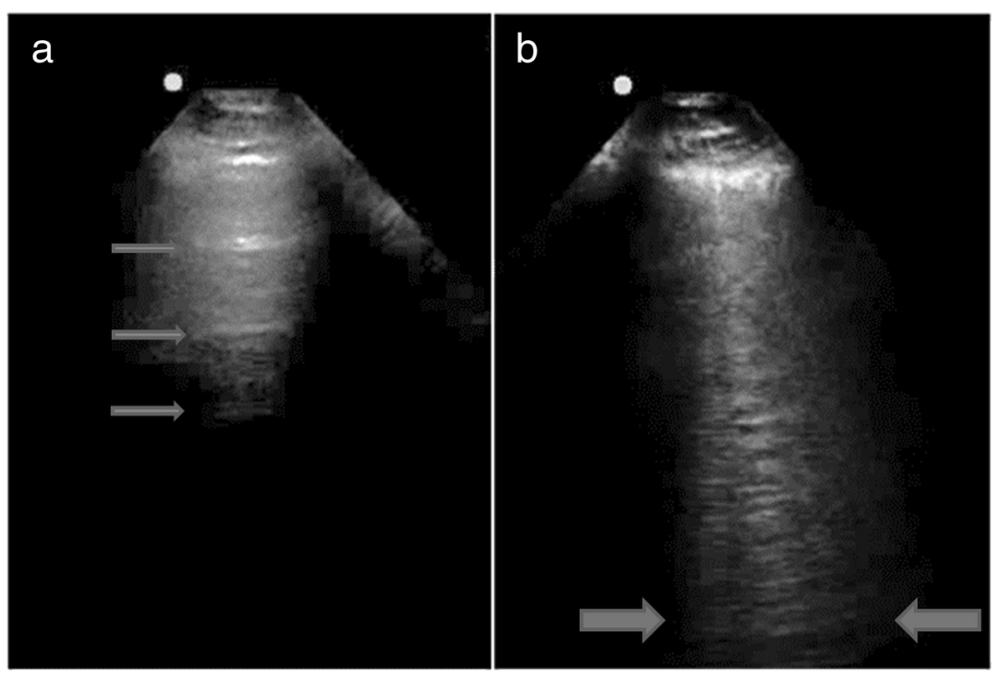

Fig. 3 a "A lines": distinct horizontal reflections in a patient with normal lungs (arrows). b "B lines": three vertical lines in a single frame extending from the pleura to the bottom of the screen in a patient with ultrasound interstitial syndrome (between arrows) 
curve (AUC). We treated each assessment as independent in our primary analysis based on frequent changes in individual patients' clinical status over 3-day intervals, but we also performed a sensitivity analysis restricted to initial assessments. STATA v11.2 (College Station, TX, USA) was used for statistical analyses.

\section{Human subjects}

This study was approved by the University of Washington Institutional Review Board. Informed consent was obtained from patients or their designee.

\section{Results}

\section{Patients and study assessments}

One hundred and twenty three study assessments were conducted on 77 patients, all of whom were mechanically ventilated and on PEEP of at least $5 \mathrm{cmH}_{2} \mathrm{O}$. The characteristics of the study patients and assessments are given in Table 1 . The median age of patients was 56 years (IQR 41-67); 52 (68\%) were male, and 24 patients underwent more than one assessment. Eight (33\%) were reclassified with respect to oxygenation criteria for ARDS and 9 (38\%) were reclassified with respect to radiographic criteria for ARDS over serial assessments. These changes included both reclassification as meeting criteria and reclassification as no longer meeting criteria. As patients were assessed no more frequently than every 3 days and because their clinical status often changed significantly in that time period, we chose assessments as our unit of analysis, treating them as independent. The most common diagnoses at the time of assessments were sepsis $(n=36,29 \%)$ and trauma $(n=31,25 \%)$.

Relationship between $\mathrm{SpO}_{2} / \mathrm{FiO}_{2}$ and $\mathrm{PaO}_{2} / \mathrm{FiO}_{2}$

At the time of ultrasound the median $\mathrm{FiO}_{2}$ was 0.40 (IQR 0.30-0.40) and the median $\mathrm{SpO}_{2}$ was 98 \% (IQR 96-100). At the time of $\mathrm{ABG}$, the median $\mathrm{FiO}_{2}$ was 0.40 (IQR 0.30-0.50), median $\mathrm{SpO}_{2}$ was 99 \% (IQR 97-100), and median $\mathrm{PaO}_{2}$ was 100 (IQR 78-122). The $\mathrm{SpO}_{2}$ at the time of $\mathrm{ABG}$ was used for all further analyses. The relationship between $\mathrm{SpO}_{2} / \mathrm{FiO}_{2}$ and $\mathrm{PaO}_{2} / \mathrm{FiO}_{2}$ derived by Rice et al. in a cohort of ARDS patients indicated that $\mathrm{SpO}_{2} / \mathrm{FiO}_{2}$ threshold values of 315 and 235 corresponded to $\mathrm{PaO}_{2} / \mathrm{FiO}_{2}$ of 300 and 200, respectively, when $\mathrm{SpO}_{2} \leq 97 \%$ [5]. To validate this relationship in our cohort of mechanically ventilated patients, we restricted our analysis to the 44 observations where $\mathrm{SpO}_{2} \leq 97 \%$ at the time of ABG. In this subset, the median $\mathrm{PaO}_{2} / \mathrm{FiO}_{2}$ was 198 (IQR 155-249) and the median $\mathrm{SpO}_{2} / \mathrm{FiO}_{2}$ was 240 (IQR 191-243). The Spearman rank correlation coefficient between $\mathrm{PaO}_{2} / \mathrm{FiO}_{2}$ and $\mathrm{SpO}_{2} /$ $\mathrm{FiO}_{2}$ was $0.74(p<0.0001)$. We identified one outlier that was characterized by marked discordance between $\mathrm{PaO}_{2}$ and $\mathrm{SpO}_{2}$ due to rapidly dynamic changes in oxygenation
Table 1 Baseline characteristics of subjects and study assessments

\begin{tabular}{|c|c|c|}
\hline Characteristic & Number & Percent \\
\hline Patients & 77 & \\
\hline Male & 52 & 68 \\
\hline Age, median (IQR) & $56(41-67)$ & \\
\hline Number undergoing 2 assessments & 13 & 17 \\
\hline Number undergoing 3 assessments & 3 & 4 \\
\hline Number undergoing $\geq 4$ assessments & 8 & 10 \\
\hline Assessments & 123 & \\
\hline \multicolumn{3}{|l|}{ Site: } \\
\hline $\mathrm{MICU}$ & 45 & 37 \\
\hline SICU & 49 & 40 \\
\hline $\mathrm{NICU}$ & 29 & 24 \\
\hline \multicolumn{3}{|l|}{ Diagnosis: } \\
\hline Sepsis & 36 & 29 \\
\hline Trauma & 31 & 25 \\
\hline Postsurgery & 24 & 20 \\
\hline CVA & 21 & 17 \\
\hline Cardiogenic pulmonary edema & 21 & 17 \\
\hline Pneumonia & 20 & 16 \\
\hline PEA or VF arrest & 13 & 11 \\
\hline PE & 10 & 8 \\
\hline ARDS & 10 & 8 \\
\hline Overdose & 9 & 7 \\
\hline Seizure & 7 & 6 \\
\hline Pancreatitis & 7 & 6 \\
\hline COPD & 6 & 5 \\
\hline $\mathrm{FiO}_{2}$ at time of $\mathrm{ABG}$, median (IQR) & $0.40(0.30-0.50)$ & \\
\hline $\mathrm{PaO}_{2} / \mathrm{FiO}_{2}$, median (IQR) & $250(180-337)$ & \\
\hline $\mathrm{SpO}_{2} \%$ at time of $\mathrm{ABG}$, median (IQR) & $99(97-100)$ & \\
\hline Bilateral opacities on CXR & 42 & 34 \\
\hline
\end{tabular}

Patients may have more than one diagnosis at the time of assessment. $A B G$ arterial blood gas, ARDS acute respiratory distress syndrome, COPD chronic obstructive pulmonary disease, CVA Cerebrovascular Accident, CXR chest $x$-ray, IQR interquartile range, MICU Medical Intensive Care Unit, NICU Neurological Intensive Care Unit, PE Pulmonary Embolism, PEA Pulseless Electrical Activity, SICU Surgical Intensive Care Unit, SpO2 pulse oximetric oxygen saturation, VF Ventricular Fibrillation

at the time of the study. Excluding this observation, the correlation between $\mathrm{PaO}_{2} / \mathrm{FiO}_{2}$ and $\mathrm{SpO}_{2} / \mathrm{FiO}_{2}$ increased to $0.83(p<0.0001)$ (Fig. 4). We performed receiver operating curve analysis to further evaluate the $\mathrm{SpO}_{2} / \mathrm{FiO}_{2}$ ratio. $\mathrm{SpO}_{2} / \mathrm{FiO}_{2}$ ratio had modest ability to discriminate $\mathrm{PaO}_{2} / \mathrm{FiO}_{2} \leq 300$, based on an AUC value of $0.76(95 \% \mathrm{CI}$ 0.34-1.00) (Additional file 1: Figure S1A). The discriminatory ability for $\mathrm{SpO}_{2} / \mathrm{FiO}_{2}$ in classifying $\mathrm{PaO}_{2} / \mathrm{FiO}_{2} \leq 200$, however, was considerably better, with an AUC of 0.89 (95 \% CI 0.80-0.99) (Additional file 1: Figure S1B). The sensitivity of $\mathrm{SpO}_{2} / \mathrm{FiO}_{2} \leq 315$ for $\mathrm{PaO}_{2} / \mathrm{FiO}_{2} \leq 300$ was 83 \% (95\% CI 68-93), and the specificity was 50 \% (95\% 


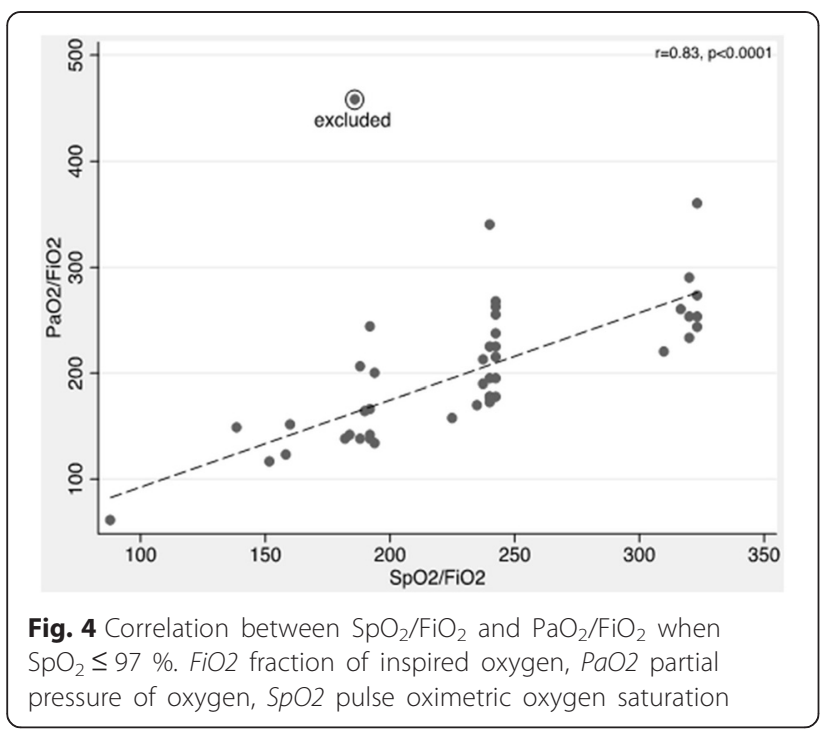

CI 1-99) (Table 2). The sensitivity of $\mathrm{SpO}_{2} / \mathrm{FiO}_{2} \leq 235$ for $\mathrm{PaO}_{2} / \mathrm{FiO}_{2} \leq 200$ was $70 \%$ (95\% CI 47-87), and the specificity was $90 \%$ (95\% CI 68-99).

\section{UIS as a marker for radiographic opacities consistent with ARDS}

Of the 738 lung fields evaluated by ultrasound (six fields for 123 assessments), 357 (48 \%) demonstrated B line predominance as interpreted by the study physician. B lines were more common in posterior lung fields but were distributed equally on the left and on the right (Fig. 5). One hundred and one ultrasound assessments were conducted within $8 \mathrm{~h}$ of a chest radiograph. We used this subset of assessments to evaluate optimal thresholds of UIS for determination of radiographic criteria of ARDS as various thresholds have been reported [7, 10-12, 15, 18]. In 35 (35 \%) assessments, bilateral

Table 2 Performance of $\mathrm{SpO}_{2} / \mathrm{FiO}_{2}$ as a marker of $\mathrm{PaO}_{2} / \mathrm{FiO}_{2}$ when $\mathrm{SpO}_{2} \leq 97 \%$

\begin{tabular}{|c|c|c|c|c|c|c|}
\hline \multicolumn{4}{|c|}{ Number of observations $(n=43)$} & \multicolumn{2}{|c|}{ Test characteristic } & \multirow[t]{2}{*}{$95 \% \mathrm{Cl}$} \\
\hline & & $\mathrm{PaO}_{2} / \mathrm{F}$ & & & & \\
\hline & & $\leq 300$ & $>300$ & Sensitivity & $83 \%$ & $68-93$ \\
\hline \multirow[t]{5}{*}{$\mathrm{SpO}_{2} / \mathrm{FiO}_{2}$} & $\leq 315$ & 34 & 1 & Specificity & $50 \%$ & $1-99$ \\
\hline & $>315$ & 7 & 1 & PPV & $97 \%$ & $85-100$ \\
\hline & & & & NPV & $13 \%$ & $0-53$ \\
\hline & & $\mathrm{PaO}_{2} / \mathrm{F}$ & & & & \\
\hline & & $\leq 200$ & $>200$ & Sensitivity & $70 \%$ & $47-87$ \\
\hline \multirow[t]{3}{*}{$\mathrm{SpO}_{2} / \mathrm{FiO}_{2}$} & $\leq 235$ & 16 & 2 & Specificity & $90 \%$ & $68-99$ \\
\hline & $>235$ & 7 & 18 & PPV & $89 \%$ & $65-99$ \\
\hline & & & & NPV & $72 \%$ & $51-88$ \\
\hline
\end{tabular}

$\mathrm{Cl}$ confidence interval, $\mathrm{FiO}_{2}$ fraction of inspired oxygen, $\mathrm{NPV}$ negative predictive value, $\mathrm{PaO}_{2}$ partial pressure of oxygen, $\mathrm{PPV}$ positive predictive value, $\mathrm{SpO} \mathrm{O}_{2}$ pulse oximetric oxygen saturation

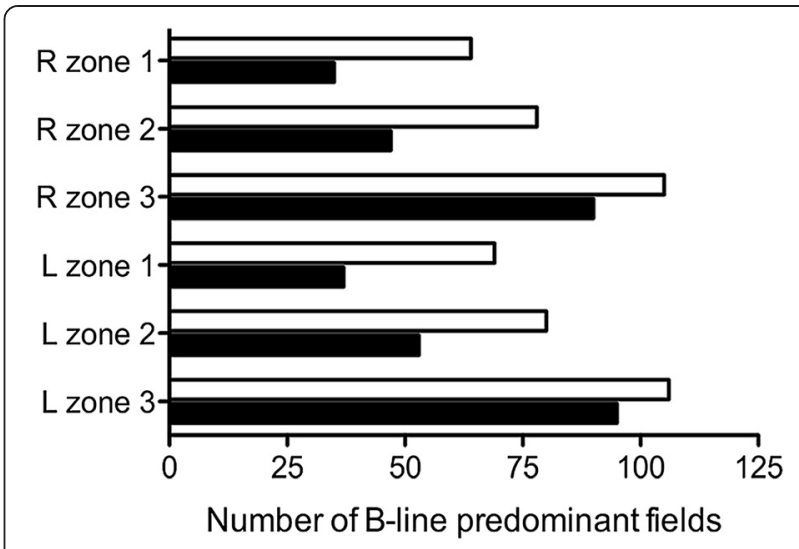

Fig. 5 Distribution of B line-predominant lung fields. Black bars indicate reads by the study physician; white bars indicate reads by the independent physician. For right $(R)$ and left $(L)$, zones correspond to locations shown in Fig. 2

opacities consistent with ARDS were apparent on chest radiograph. The sensitivity and specificity of UIS in at least one lung field bilaterally (UIS-2) for radiographic ARDS were $86 \%$ (95\% CI 70-95) and $38 \%$ (95\% CI 26-51) (Table 3). The sensitivity and specificity of UIS in at least one field bilaterally and involving a minimum of three lung fields (UIS-3) were $80 \%$ (95\% CI 63-92) and $62 \%$ (95\% CI 49-74). The sensitivity and specificity of UIS in at least two lung fields bilaterally (UIS-4) were $60 \%$ (95\% CI 42-76) and 77 \% (95 \% CI 65-87). By receiver operating curve analysis, the ability of UIS pattern to discriminate radiographic ARDS was fair (AUC 0.73, 95 \% CI 0.63-0.83) (Additional file 2: Figure S2).

\section{Interobserver reliability of UIS interpretation}

To test interobserver reliability of the ultrasound interpretations, all ultrasound videos were independently reviewed by an ultrasound-trained attending physician who had not participated in training or data collection and who was blinded to the clinical scenario. The kappa coefficient between the two interpreters for designating $\mathrm{B}$ lines present in a lung field was 0.57 (CI 0.40-0.73), consistent with moderate agreement. In comparison to the study physician, the independent physician classified a higher proportion of lung fields as B line-predominant but the relative distribution of $B$ lines in the left lung compared to right lung, and throughout the upper, middle, and lower lung zones was comparable (Fig. 5). We evaluated the interobserver reliability for different UIS thresholds. For UIS-2, the kappa coefficient was 0.44 (CI 0.05-0.83); for UIS-3, the kappa coefficient was 0.45 (CI 0.09-0.81); and for UIS-4, the kappa coefficient was 0.47 (CI 0.07-0.87). Using the independent physician's classifications, sensitivity and specificity of UIS-2 for radiographic criteria of ARDS were $89 \%$ (95\% CI 73-97) and $15 \%$ (95\% CI 8-26), respectively; $89 \%$ (95\% CI 73-97) and 29 \% (95 \% CI 18-41) for UIS-3; and 
Table 3 Performance of ultrasound diagnosis of ultrasound interstitial syndrome (UIS) as a marker of bilateral pulmonary opacities consistent with acute respiratory distress syndrome

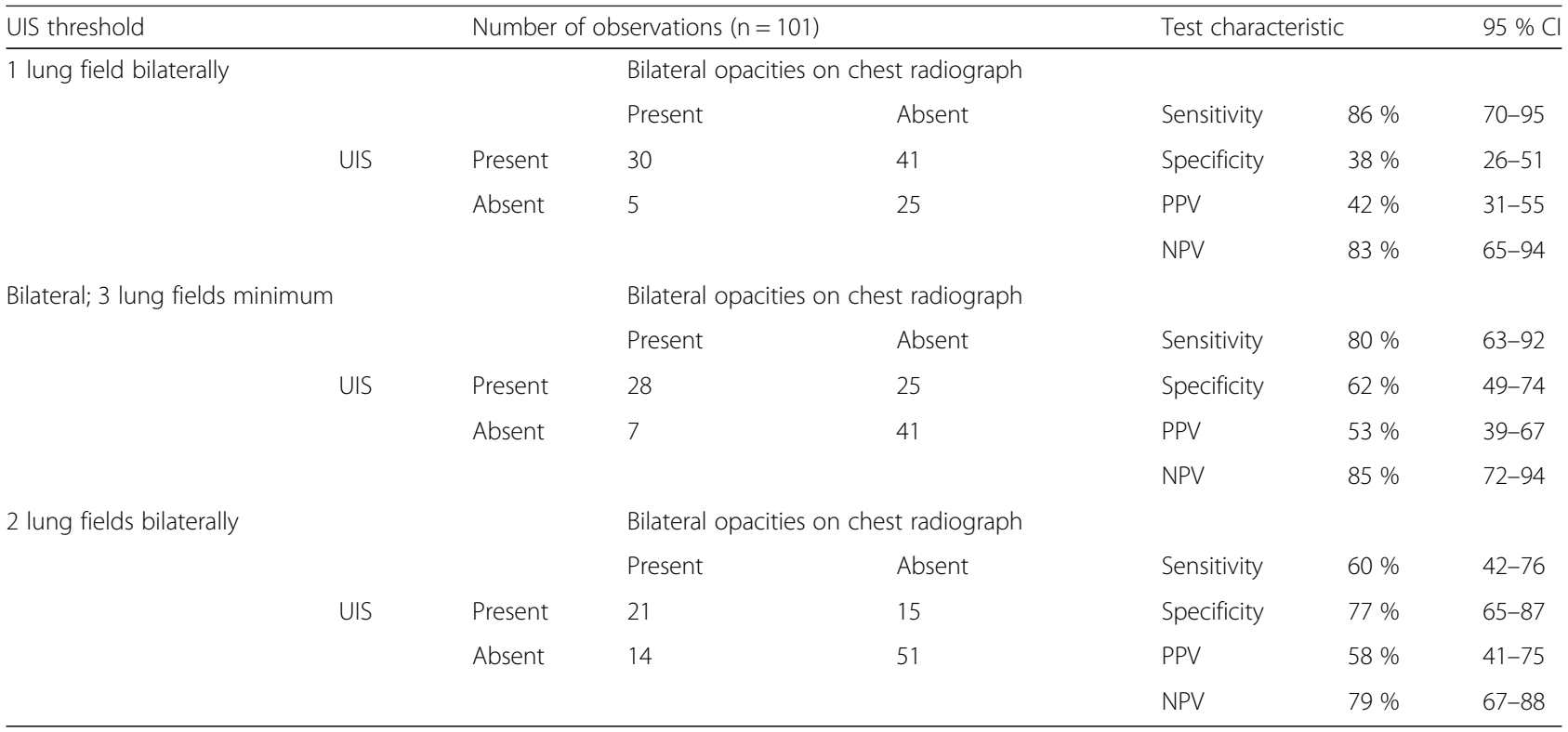

Cl confidence interval, NPV negative predictive value, PPV positive predictive value

$83 \%$ (95 \% CI 66-93) and $46 \%$ (95\% CI 33-58) for UIS-4 (Table 3).

\section{$\mathrm{SpO}_{2} / \mathrm{FiO}_{2}$ and UIS as marker for oxygenation and radiographic criteria for ARDS}

We next evaluated the combination of pulse oximetry measurements and pulmonary ultrasound assessments as markers for the coexistence of oxygenation and radiographic criteria for ARDS in our cohort. We combined $\mathrm{SpO}_{2} / \mathrm{FiO}_{2} \leq 315$ and ultrasound demonstrating UIS-3 in the subset of 33 observations with $\mathrm{SpO}_{2} \leq 97 \%$, a chest radiograph within $8 \mathrm{~h}$ of ultrasound, and ABG within $24 \mathrm{~h}$ of ultrasound (Table 4). The sensitivity of the combination of pulse oximetry and ultrasound determinations of oxygenation and radiographic ARDS criteria was $83 \%$ (95\% CI 52-98) and specificity was $62 \%$ (95\% CI $38-82)$. The positive predictive value was $56 \%$ (95\% CI $31-79)$ and the negative predictive value was $87 \%$ (95\% CI 60-98). We repeated this analysis using a threshold $\mathrm{SpO}_{2} / \mathrm{FiO}_{2} \leq 235$, restricting to cases of at least moderate ARDS $\left(\mathrm{PaO}_{2} / \mathrm{FiO}_{2} \leq 200\right)$ and found that the sensitivity was $64 \%(95 \%$ CI 31-89) and specificity was $86 \%$ (95\% CI 65-97). The positive predictive value was $70 \%$ (95\% CI 35-93) and the negative predictive value was $83 \%$ (95 \% CI 61-95).

We considered the ultrasound interpretations of the independent physician and repeated these analyses. The sensitivity of the combination of pulse oximetry $\left(\mathrm{SpO}_{2} /\right.$ $\mathrm{FiO}_{2} \leq 315$ ) and ultrasound (UIS-3) for oxygenation and radiographic ARDS criteria was $91 \%$ (95 \% CI 62-100) and specificity was $48 \%$ (95\% CI 26-70) (Table 4). The sensitivity of the combination of pulse oximetry $\left(\mathrm{SpO}_{2} /\right.$ $\mathrm{FiO}_{2} \leq 235$ ) and ultrasound (UIS-3) for oxygenation and radiographic criteria of severe ARDS was $73 \%$ (95\% CI 39-94) and specificity was $77 \%$ (95 \% CI 55-92).

\section{Sensitivity to repeat assessments}

To determine whether our treatment of each assessment as independent altered our findings, we performed sensitivity analyses restricted to the first assessment for each of the 77 patients. We did not find any significant differences in the relationships between $\mathrm{SpO}_{2} / \mathrm{FiO}_{2}$ and $\mathrm{PaO}_{2} /$ $\mathrm{FiO}_{2}$. Diagnostic accuracy of UIS as a marker for radiographic opacities consistent with ARDS was also similar: the sensitivity and specificity of UIS-3 were $80 \%$ (95\% CI 56-94) and $72 \%$ (95\% CI 57-84). The sensitivity of the combination of pulse oximetry and ultrasound determinations of oxygenation and radiographic ARDS criteria $\left(\mathrm{SpO}_{2} / \mathrm{FiO}_{2} \leq 315\right.$ and UIS-3) was $88 \%(95 \% \mathrm{CI}$ 47-100) and specificity was $69 \%$ (95 \% CI 39-91).

\section{Discussion}

The purpose of this pilot study was to assess the performance of rapid assessment with bedside pulmonary ultrasound and use of pulse oximetry as alternatives to chest radiograph and ABG in the diagnosis of ARDS. The results of this study showed that, in mechanically ventilated ICU patients, $\mathrm{SpO}_{2} / \mathrm{FiO}_{2}$ and $\mathrm{PaO}_{2} / \mathrm{FiO}_{2}$ are highly correlated, that $\mathrm{SpO}_{2} / \mathrm{FiO}_{2} \leq 315$ is quite sensitive for $\mathrm{PaO}_{2} / \mathrm{FiO}_{2} \leq 300$, and that $\mathrm{SpO}_{2} / \mathrm{FiO}_{2} \leq 235$ is highly specific for $\mathrm{PaO}_{2} / \mathrm{FiO}_{2} \leq 200$. Our study confirms reasonable sensitivity of simplified six-point lung ultrasound in 
Table 4 Performance of $\mathrm{SpO}_{2} / \mathrm{FiO}_{2}$ and ultrasound interstitial syndrome (UIS) as a marker for with acute respiratory distress syndrome (ARDS) criteria when $\mathrm{SpO}_{2} \leq 97 \%$

\begin{tabular}{|c|c|c|c|c|c|c|}
\hline & & Number of observations $(n=33)$ & & Test charact & & $95 \% \mathrm{Cl}$ \\
\hline \multirow[t]{2}{*}{ Study physician } & & \multicolumn{5}{|c|}{ Oxygenation and radiographic criteria for ARDS } \\
\hline & & Present & Absent & Sensitivity & $83 \%$ & $52-98$ \\
\hline \multirow[t]{5}{*}{$\mathrm{SpO}_{2} / \mathrm{FiO}_{2} \leq 315$ and UIS } & Present & 10 & 8 & Specificity & $62 \%$ & $38-82$ \\
\hline & Absent & 2 & 13 & PPV & $56 \%$ & $31-79$ \\
\hline & & & & NPV & $87 \%$ & $60-98$ \\
\hline & & \multicolumn{5}{|c|}{ Oxygenation and radiographic criteria for moderate-severe ARDS } \\
\hline & & Present & Absent & Sensitivity & $64 \%$ & $31-89$ \\
\hline \multirow[t]{3}{*}{$\mathrm{SpO}_{2} / \mathrm{FiO}_{2} \leq 235$ and UIS } & Present & 7 & 3 & Specificity & $86 \%$ & $65-97$ \\
\hline & Absent & 4 & 19 & PPV & $70 \%$ & $35-93$ \\
\hline & & & & NPV & $83 \%$ & $61-95$ \\
\hline \multirow[t]{2}{*}{ Independent physician } & & \multicolumn{5}{|c|}{ Oxygenation and radiographic criteria for ARDS } \\
\hline & & Present & Absent & Sensitivity & $91 \%$ & $62-100$ \\
\hline \multirow[t]{5}{*}{$\mathrm{SpO}_{2} / \mathrm{FiO}_{2} \leq 315$ and UIS } & Present & 11 & 11 & Specificity & $48 \%$ & $26-70$ \\
\hline & Absent & 1 & 10 & PPV & $50 \%$ & $28-72$ \\
\hline & & & & NPV & $91 \%$ & $59-100$ \\
\hline & & Oxygenation and radiographic $\mathrm{c}$ & ia for moderate-severe ARDS & & & \\
\hline & & Present & Absent & Sensitivity & $73 \%$ & 39-94 \\
\hline \multirow[t]{3}{*}{$\mathrm{SpO}_{2} / \mathrm{FiO}_{2} \leq 235$ and UIS } & Present & 8 & 5 & Specificity & $77 \%$ & 55-92 \\
\hline & Absent & 3 & 17 & PPV & $62 \%$ & $32-86$ \\
\hline & & & & NPV & $85 \%$ & $62-97$ \\
\hline
\end{tabular}

UIS defined as 3 or more B lines bilaterally and involving a minimum of three lung fields. $\mathrm{Cl}$ confidence interval, $\mathrm{FiO}_{2}$ fraction of inspired oxygen, $\mathrm{NPV} \mathrm{negative}$ predictive value, $\mathrm{PaO}_{2}$ partial pressure of oxygen, $\mathrm{PPV}$ positive predictive value, $\mathrm{SpO}_{2}$ pulse oximetric oxygen saturation

identifying patients with bilateral pulmonary opacities consistent with ARDS on chest radiograph using a threshold of bilateral UIS involving at least three lung fields in total, although specificity was lower. Finally, our data on a relatively small number of patients indicate that the combination of $\mathrm{SpO}_{2} / \mathrm{FiO}_{2} \leq 315$ and bilateral/3 field UIS on ultrasound is sensitive for the classification of traditional oxygenation and radiographic criteria for ARDS in mechanically ventilated patients; in contrast, the combination of $\mathrm{SpO}_{2} / \mathrm{FiO}_{2} \leq 235$ and bilateral/3 field UIS on ultrasound is specific for moderate-severe ARDS.

Overall, we found that the $\mathrm{SpO}_{2} / \mathrm{FiO}_{2}$ cutoffs established by Rice et al. were less predictive of the $\mathrm{PaO}_{2} /$ $\mathrm{FiO}_{2}$ than originally described [5]. Rice et al. found higher sensitivity in their study, with $\mathrm{SpO}_{2} / \mathrm{FiO}_{2} \leq 235$ resulting in $85 \%$ sensitivity with $85 \%$ specificity for $\mathrm{PaO}_{2} / \mathrm{FiO}_{2} \leq 200$, and $91 \%$ sensitivity with $56 \%$ specificity of $\mathrm{SpO}_{2} / \mathrm{FiO}_{2} \leq 315$ to predict $\mathrm{PaO}_{2} / \mathrm{FiO}_{2} \leq 300$. Several explanations may account for this. First, the use of the $\mathrm{SpO}_{2} / \mathrm{FiO}_{2}$ ratio is limited by flattening of the oxyhemoglobin dissociation curve at high $\mathrm{SpO}_{2}$; this effect is exacerbated when $\mathrm{FiO}_{2}$ is not maximally reduced. Many patients in our study were administered a "minimum" $\mathrm{FiO}_{2}$ of 0.4 . Rice et al. report that their studies targeted $\mathrm{SpO}_{2}$ values between 92 and $94 \%$, whereas very few of our subjects had $\mathrm{SpO}_{2}<97 \%$. Second, Rice et al. analyzed 1,076 patients enrolled in ARDS studies; this contrasts markedly with our smaller, relatively unfiltered cohort of 77 mechanically ventilated patients. Third, our study is a single-center observational study, in contrast to the multicenter interventional ARDSNet studies. Future studies refining test characteristics of new ARDS criteria should determine $\mathrm{SpO}_{2} / \mathrm{FiO}_{2}$ at the lowest possible $\mathrm{FiO}_{2}$, which would require cooperation with respiratory therapists and nursing staff.

The sensitivity and specificity of ultrasound assessments of UIS for radiographic criteria of ARDS in our study were also less than was predicted based on prior studies. There are several possible reasons for this. The original diagnostic algorithm for the BLUE protocol includes an assessment of the lung sliding by ultrasound [10]. This specific criterion was removed from our study, as in trauma patients with chest injuries one might expect a loss of lung sliding without ruling out ARDS or cardiogenic pulmonary edema. An additional challenge in the trauma patient is the evaluation in the setting of significant subcutaneous emphysema. In this setting ultrasound images are difficult to interpret, and may provide false reassurance to novice sonographers simply counting B lines or looking for hepatization. A phased- 
array probe was used in this study instead of the micro convex probe used in many other studies. This was chosen based on an assessment that the two probes most likely to be found in a resource limited setting were a linear probe (for superficial assessments and procedures) and a phased-array probe (for cardiac, intraabdominal, and obstetric assessment). In addition, unlike other studies $[10,11,18]$, our study used only chest radiographs and not chest computed tomography imaging to determine radiographic criteria for ARDS.

About a third of ultrasound assessments we performed had at least one lung field for which the imaging clip was considered difficult to interpret. Specifically, trauma patients with subcutaneous emphysema and supine obese patients with significant distance to the lung tissue represent a technical challenge. The division of the chest into six zones as performed in the BLUE protocol and the ICU-SOUND protocol allows rapid assessment of anterior and posterior-lateral fields $[15,18]$. Other studies have utilized more lung fields, with the international consensus statement specifying an eight-zone protocol published by Volpicelli et al. [7]. Our study was designed as a rapid diagnostic tool leading to a binary outcome, but the diagnosis of ARDS in other ultrasound studies was one of several potential outcomes at the end of a diagnostic algorithm. By distilling the process to simply an "A-line" or "B-line" determination for each lung field, much of the information we acquired in the process of ultrasound was disregarded, including the presence or absence of lung sliding and images that showed clear signs of consolidation, hepatization or effusions. In the BLUE protocol these findings would have potentially changed the final ultrasound-based diagnosis, and likely contributed to the moderate interobserver agreement we observed. Six points of examination may also not be sufficient for clear identification of alternative processes, as the study that most accurately identified ARDS using pulmonary ultrasound did so by scanning each intercostal space [11].

Alternative methods for ascertainment of imaging and oxygenation criteria for ARDS may be useful in a variety of settings where critically ill patients are managed. Ultrasound evaluation may be faster and offer additional benefits compared to chest radiography [12, 19]. In lowresource settings without portable chest radiography and ABG testing capacity, pulse oximeters and ultrasound machines are increasingly available [3, 4]. Ultrasound is a useful imaging modality in these settings due to its versatility and portability. Moreover, dependence on traditional tools for diagnosing ARDS in low-resource settings may substantially underestimate the incidence of disease [2]. The diagnosis of ARDS is important even when resources are limited because two of the management strategies demonstrated to improve mortality in
ARDS - lung protective ventilation and proning [20, 21] - are cheap and potentially feasible to implement in a range of settings. While some evidence supports more liberal use of lung protective ventilation in respiratory failure [22], understanding the prevalence of ARDS is one element in a necessary effort to improve detection and treatment of respiratory diseases and critical illness in low-resource settings globally [23-27].

Our study offers several strengths. First, our study was conducted by a novice sonographer quickly and without moving the patient. While several other studies have examined the ability of ultrasound to identify findings consistent among patients with ARDS [10-12, 18], conditions were optimized: patients were positioned carefully and expert sonographers obtained multiple ultrasound findings in combination. Thus our study was pragmatic and modeled "real-world" conditions for busy ICU practitioners. Second, we methodically evaluated a sequentially enrolled cohort of critically ill medical and surgical patients at risk for ARDS in a large referral center, suggesting external validity of our study to other busy critical care centers. Third, we enrolled patients with a range of diseases and $\mathrm{PaO}_{2} / \mathrm{FiO}_{2}$ ratios; thus our tests of diagnostic accuracy measures should apply to similar spectra of disease. Fourth, we carefully considered the timing of various diagnostic tests in relation to each study observation in order to minimize effects of temporal changes in clinical condition on the analysis. Fifth, although we treated each assessment as independent, we confirmed that intraindividual correlation did not alter our findings. Finally, we tested interobserver effects by performing a secondary analysis of ultrasound characteristics using an independent ultrasound-trained physician to classify images.

Our study also has several limitations. Most notably, this was a small, single-center study with ultrasound data obtained by only one sonographer. Furthermore, few patients had $\mathrm{SpO}_{2}<97 \%$, limiting the number of observations that could be analyzed according to the methodology of Rice et al. [5]. As this was an observational study, additional patients were excluded from analysis if the duration of time between their radiograph or ABG and the study observation was too long. These restrictions resulted in a small population for final analyses. In addition, if less ill patients had less frequent diagnostic tests, our analyses may have been biased towards sicker patients. While the study physician was blinded to the clinical picture for initial assessments, repeat assessments were performed after medical record review, potentially leading to bias. Evaluating only intubated patients limits the generalizability of our results, particularly to lower resourced settings. As noted above, a six-point protocol may be insufficient and a binary "B-line predominant" determination may result in 
disregarding potentially important clinical information. Avoiding patient repositioning meant that there was limited visualization of the posterior lung fields, which is particularly relevant as ARDS is a posterior predominant condition. Potentially, diagnostic yield would have been higher had assistance been sought. Furthermore, chest radiography is a suboptimal gold standard when compared to chest computed tomography imaging [12].

\section{Conclusions}

The combination of pulse oximetry and six-point rapid bedside pulmonary ultrasound assessment provides a reasonably sensitive method for identifying intubated patients who meet standard ARDS oxygenation and imaging criteria. Future, larger studies in high- and lowresource settings are needed to validate and refine the utility of these modalities in diagnosing lung disease in critically ill patients in under-resourced settings.

\section{Key messages}

- In this pilot study of ICU patients receiving invasive mechanical ventilation, a simplified six-point pulmonary ultrasound assessment for "B lines" is sensitive in identifying bilateral opacities on chest radiography consistent with ARDS.

- Pulse oximetry-derived $\mathrm{SpO}_{2} / \mathrm{FiO}_{2} \leq 315$ is a sensitive threshold for $\mathrm{PaO}_{2} / \mathrm{FiO}_{2} \leq 300$; pulse oximetry-derived $\mathrm{SpO}_{2} / \mathrm{FiO}_{2} \leq 235$ is a specific threshold for $\mathrm{PaO}_{2} / \mathrm{FiO}_{2} \leq 200$.

- The combination of pulmonary ultrasound assessment and $\mathrm{SpO}_{2} / \mathrm{FiO}_{2}$ measurement provides a reasonably sensitive method to identify patients who meet conventional ARDS oxygenation and imaging criteria.

- Based on this pilot investigation, further studies to quantify the performance of alternative ultrasound protocols to identify conditions such as ARDS are warranted.

\section{Additional files}

Additional file 1: Figure S1. Receiver operating curves for $\mathrm{SpO}_{2} / \mathrm{FiO}_{2}$ ratio in discriminating $\mathrm{PaO}_{2} / \mathrm{FiO}_{2} \leq 300$ (A) and in discriminating $\mathrm{PaO}_{2} /$ $\mathrm{FiO}_{2} \leq 200$ (B) when $\mathrm{SpO}_{2} \leq 97 \%$.

Additional file 2: Figure S2. Receiver operating curve for UIS pattern in discriminating bilateral opacities consistent with ARDS on chest radiograph. Three thresholds for UIS were defined: B lines in at least one lung field bilaterally, in at least one field bilaterally and involving a minimum of three lung fields, or in at least two lung fields bilaterally.

\section{Abbreviations}

ABG: Arterial blood gas; ARDS: Acute respiratory distress syndrome; AUC: Area under the curve; Cl: Confidence interval; CPAP: Continuous positive airway pressure; $\mathrm{FiO}_{2}$ : Fraction of inspired oxygen; IQR: Interquartile range; $\mathrm{PaO}_{2}$ : Partial pressure of oxygen; PEEP: Positive end-expiratory pressure; $\mathrm{SpO}_{2}$ : Pulse oximetric oxygen saturation; UIS: Ultrasound interstitial syndrome.

\section{Competing interests}

The authors declare that they have no competing interests.

\section{Authors' contributions}

CMB, DRS and TEW participated in the design of the study. CMB performed all ultrasound assessments and acquired data. CMB and AAA interpreted all ultrasound assessments. CMB and TEW performed statistical analysis and drafted the manuscript. All authors read and approved the final manuscript.

\section{Acknowledgments}

We thank the ICU staff at Harborview Medical Center who facilitated this study, Dr Sudhakar Pipavath for chest radiograph interpretation, and Dr Chris Goss for critical review of the manuscript.

Received: 4 March 2015 Accepted: 26 June 2015

Published online: 21 July 2015

\section{References}

1. Bernard GR, Artigas A, Brigham KL, Carlet J, Falke K, Hudson L, et al. The American-European Consensus Conference on ARDS. Definitions, mechanisms, relevant outcomes, and clinical trial coordination. Am J Respir Crit Care Med. 1994;149:818-24.

2. Riviello E, Talmor D, Novack V, Kiviri W, Twagirumugabe T, BannerGoodspeed V, et al. Incidence and outcomes of ARDS in Rwanda using modified Berlin criteria for resource-poor settings. Crit Care Med. 2014;42:A1447-8.

3. Sippel S, Muruganandan K, Levine A, Shah S. Review article: use of ultrasound in the developing world. Int J Emerg Med. 2011;4:72

4. Funk LM, Weiser TG, Berry WR, Lipsitz SR, Merry AF, Enright AC, et al. Global operating theatre distribution and pulse oximetry supply: an estimation from reported data. Lancet. 2010;376:1055-61.

5. Rice TW, Wheeler AP, Bernard GR, Hayden DL, Schoenfeld DA, Ware LB, Comparison of the $\mathrm{SpO} 2 / \mathrm{FIO} 2$ ratio and the $\mathrm{PaO} 2 / \mathrm{FIO} 2$ ratio in patients with acute lung injury or ARDS. Chest. 2007;132:410-7.

6. Khemani RG, Patel NR, Bart 3rd RD, Newth CJ. Comparison of the pulse oximetric saturation/fraction of inspired oxygen ratio and the $\mathrm{PaO} /$ /fraction of inspired oxygen ratio in children. Chest. 2009;135:662-8.

7. Volpicelli G, Elbarbary M, Blaivas M, Lichtenstein DA, Mathis G, Kirkpatrick AW, et al. International evidence-based recommendations for point-of-care lung ultrasound. Intensive Care Med. 2012;38:577-91.

8. Lichtenstein D, Meziere G, Biderman P, Gepner A. The comet-tail artifact An ultrasound sign of alveolar-interstitial syndrome. Am J Respir Crit Care Med. 1997;156:1640-6.

9. Volpicelli G, Mussa A, Garofalo G, Cardinale L, Casoli G, Perotto F, et al. Bedside lung ultrasound in the assessment of alveolar-interstitial syndrome. Am J Emerg Med. 2006:24:689-96.

10. Lichtenstein DA, Meziere GA. Relevance of lung ultrasound in the diagnosis of acute respiratory failure: the BLUE protocol. Chest. 2008;134:117-25.

11. Copetti R, Soldati G, Copetti P. Chest sonography: a useful tool to differentiate acute cardiogenic pulmonary edema from acute respiratory distress syndrome. Cardiovasc Ultrasound. 2008;6:16.

12. Lichtenstein D, Goldstein I, Mourgeon E, Cluzel P, Grenier P, Rouby JJ. Comparative diagnostic performances of auscultation, chest radiography, and lung ultrasonography in acute respiratory distress syndrome. Anesthesiology. 2004;100:9-15.

13. Bedetti G, Gargani L, Corbisiero A, Frassi F, Poggianti E, Mottola G. Evaluation of ultrasound lung comets by hand-held echocardiography. Cardiovasc Ultrasound. 2006;:34.

14. Baker K, Mitchell G, Stieler G. Limited lung ultrasound protocol in elderly patients with breathlessness; agreement between bedside interpretation and stored images as acquired by experienced and inexperienced sonologists. Australasian J Ultrasound Med. 2013;16:86-92.

15. Lichtenstein DA, Mezière GA. The BLUE-points: three standardized points used in the BLUE-protocol for ultrasound assessment of the lung in acute respiratory failure. Critical Ultrasound Journal. 2011;3:109-10.

16. Lichtenstein DA. Lung ultrasound in the critically ill. Ann Intensive Care. 2014;4:1. 
17. Ranieri VM, Rubenfeld GD, Thompson BT, Ferguson ND, Caldwell E, Fan E, et al. Acute respiratory distress syndrome: the Berlin Definition. JAMA. 2012;307:2526-33.

18. Manno E, Navarra M, Faccio L, Motevallian M, Bertolaccini L, Mfochive A, et al. Deep impact of ultrasound in the intensive care unit: the "ICU-sound" protocol. Anesthesiology. 2012;117:801-9.

19. Ashton-Cleary DT. Is thoracic ultrasound a viable alternative to conventional imaging in the critical care setting? Br J Anaesth. 2013;111:152-60.

20. Determann RM, Royakkers A, Wolthuis EK, Vlaar AP, Choi G, Paulus F, et al. Ventilation with lower tidal volumes as compared with traditional tidal volumes for acute lung injury and the acute respiratory distress syndrome. The Acute Respiratory Distress Syndrome Network. N Engl J Med. 2000;342:1301-8.

21. Guerin C, Reignier J, Richard JC, Beuret P, Gacouin A, Boulain T, et al. Prone positioning in severe acute respiratory distress syndrome. N Engl J Med. 2013:368:2159-68

22. Serpa Neto A, Cardoso SO, Manetta JA, Pereira VG, Esposito DC, Pasqualucci Mde $\mathrm{O}$, et al. Association between use of lung-protective ventilation with lower tidal volumes and clinical outcomes among patients without acute respiratory distress syndrome: a meta-analysis. JAMA. 2012;308:1651-9.

23. Einav S, Hick JL, Hanfling D, Erstad BL, Toner ES, Branson RD, et al. Surge capacity logistics: care of the critically ill and injured during pandemics and disasters: CHEST Consensus Statement. Chest. 2014;146:e17S-43.

24. Hick JL, O'Laughlin DT. Concept of operations for triage of mechanical ventilation in an epidemic. Acad Emerg Med. 2006:13:223-9.

25. Adhikari NK, Fowler RA, Bhagwanjee S, Rubenfeld GD. Critical care and the global burden of critical illness in adults. Lancet. 2010;376:1339-46.

26. Dunser MW, Baelani I, Ganbold L. A review and analysis of intensive care medicine in the least developed countries. Crit Care Med. 2006;34:1234-42.

27. Biddison LD et al. Ethical considerations: care of the critically ill and injured during pandemics and disasters: CHEST consensus statement. Chest J. 2014:146:e145S-55

\section{Submit your next manuscript to BioMed Central and take full advantage of:}

- Convenient online submission

- Thorough peer review

- No space constraints or color figure charges

- Immediate publication on acceptance

- Inclusion in PubMed, CAS, Scopus and Google Scholar

- Research which is freely available for redistribution 